\title{
The Adjunction Inequality for Weyl-Harmonic Maps
}

https://doi.org/10.1515/coma-2020-0007

Received September 20, 2019; accepted March 5, 2020

Abstract: In this paper we study an analog of minimal surfaces called Weyl-minimal surfaces in conformal manifolds with a Weyl connection $\left(M^{4}, c, D\right)$. We show that there is an Eells-Salamon type correspondence between nonvertical $\mathrm{J}$-holomorphic curves in the weightless twistor space and branched Weyl-minimal surfaces. When $(M, C, J)$ is conformally almost-Hermitian, there is a canonical Weyl connection. We show that for the canonical Weyl connection, branched Weyl-minimal surfaces satisfy the adjunction inequality

$$
\chi\left(T_{f} \Sigma\right)+\chi\left(N_{f} \Sigma\right) \leq \pm c_{1}\left(f^{\star} T^{(1,0)} M\right) .
$$

The $\pm J$-holomorphic curves are automatically Weyl-minimal and satisfy the corresponding equality. These results generalize results of Eells-Salamon and Webster for minimal surfaces in Kähler 4-manifolds as well as their extension to almost-Kähler 4-manifolds by Chen-Tian, Ville, and Ma.

Keywords: Almost-Complex Manifolds, Twistor Space, Weyl Geometry

MSC: 32Q60, 53C28, 53C43

\section{Introduction}

This paper describes an extension of the notion of minimal surfaces to the setting of conformal manifolds with a Weyl connection. Particular attention is given to almost-Hermitian 4-manifolds endowed with their canonical Weyl connection. We first review the relevant standard theory.

According to Eells and Salamon [5], branched minimal surfaces in an oriented Riemannian 4-manifold $M$ have a one-to-one correspondence with nonvertical $\mathcal{J}$-holomorphic curves in the twistor space $\mathcal{Z}$, where $\mathcal{J}$ is the canonical non-integrable almost-complex structure on $z$. Applying twistor techniques, they further show that if $M$ is almost-Kähler with almost-complex structure $J$, the $\pm J$-holomorphic curves are minimal. When $M$ is Kähler they prove the adjunction inequality,

$$
\chi\left(T_{f} \Sigma\right)+\chi\left(N_{f} \Sigma\right) \leq \pm c_{1}\left(f^{\star} T^{(1,0)} M\right),
$$

where $T_{f} \Sigma$ is the tangent bundle to $\Sigma$ ramified at the branch points of $f$, and $N_{f} \Sigma$ is its normal bundle in $f^{\star} T M$. Concurrently, Webster [15] obtained his formulas (1), (2) for a minimal surface in a Kähler 4-manifold, which imply the adjunction inequality. The adjunction inequality was extended to minimal surfaces in almostKähler 4-manifolds by Chen-Tian [3], Ville [14], and Ma [10].

This leads to the following picture for almost-Kähler manifolds: The adjunction inequality holds for minimal surfaces; every $\pm J$-holomorphic curve is minimal, and equality holds in (3) with the corresponding sign.

For an almost-Hermitian manifold, in general, the $\pm J$-holomorphic curves are not minimal, and in [3] they remark that the (3) will not hold for minimal surfaces. In this paper we show that the above scenario for almost-Kähler manifolds can be extended to almost-Hermitian manifolds when considering a conformally

^Corresponding Author: Robert Ream: Clark University Worcester, MA, United States, E-mail: rream@clarku.edu 
invariant condition on surfaces related to the minimal condition. We now briefly describe this condition and list our main theorems.

Let $M$ be a manifold with conformal metric $c$ and Weyl connection $\nabla^{D}$, to be described in detail later. For $i: \Sigma \rightarrow M$ an immersed submanifold, the Weyl second fundamental form $B$ is defined in [13] as follows. Taking $g \in c$, there is a one-form $\alpha_{g}$ such that $\nabla^{D} g=-2 \alpha_{g} \otimes g$. Let $A_{g}$ be the usual second fundamental form, then

$$
B=A_{g}-\left(\alpha_{g}^{\sharp g}\right)^{\perp} \otimes g .
$$

We say the submanifold is Weyl-minimal if $\operatorname{tr}_{i^{*} g} B=0$. Branched Weyl-minimal then has the obvious meaning.

We extend the Eells-Salamon twistor correspondence as follows.

Theorem 1.1. Let $\left(M^{4}, c, D\right)$ be a Weyl manifold, and $(\Sigma,[\eta])$ a Riemann surface. There are complex structures $J_{ \pm}$on the weightless twistor spaces $z_{ \pm}$which give a 1-to-1 correspondence between non-vertical $J_{ \pm}$-holomorphic curves $\tilde{f}_{ \pm}: \Sigma \rightarrow Z_{ \pm}$and non-constant weakly conformal branched Weyl-minimal immersions $f: \Sigma \rightarrow M$.

If the Weyl derivative is exact, then the Weyl-minimal surfaces are minimal for a preferred metric in $c$, and this is just the usual correspondence for that metric.

Theorem 1.2. Let $\left(M^{4}, c, J, D\right)$ be a conformally almost-Hermitian manifold with its canonical Weyl connection. The almost-complex structure gives rise to a $\mathcal{J}_{+}$-holomorphic section of $Z_{+}$.

This and the previous theorem imply the following corollary.

Corollary 1.1. Under the assumptions of Theorem 1.2, $a \pm J$-holomorphic curve $f: \Sigma \rightarrow M$ is a weakly conformal branched Weyl-minimal immersion.

Finally we prove that Webster's formulas hold for branched Weyl-minimal immersions.

Theorem 1.3. For a Riemann surface $(\Sigma,[\eta])$ and a conformally almost-Hermitian manifold with its canonical Weyl-connection $\left(M^{4}, c, J, D\right)$, if $f: \Sigma \rightarrow M$ is a weakly conformal branched Weyl-minimal immersion with $P$ complex points and $Q$ anti-complex points then

$$
\begin{aligned}
\chi\left(T_{f} \Sigma\right)+\chi\left(N_{f} \Sigma\right) & =-P-Q \\
c_{1}\left(f^{\star} T^{(1,0)} M\right) & =P-Q .
\end{aligned}
$$

The adjunction inequality follows from $P$ and $Q$ being positive.

Corollary 1.2. For a Riemann surface $(\Sigma,[\eta])$ and a conformally almost-Hermitian manifold with its canonical Weyl-connection $\left(M^{4}, c, J, D\right)$, if $f: \Sigma \rightarrow M$ is a weakly conformal branched Weyl-minimal immersion then

$$
\chi\left(T_{f} \Sigma\right)+\chi\left(N_{f} \Sigma\right) \leq \pm c_{1}\left(f^{\star} T^{(1,0)} M\right) .
$$

The corresponding equality holds for $\pm J$-holomorphic curves.

\section{Preliminaries}

\subsection{Weyl Geometry}

By definition, the density bundle on an $n$-dimensional manifold $M$ is $L:=\left|\Lambda^{n} T M\right|^{\frac{1}{n}}$. The tensor bundles $L^{w} \otimes T M^{j} \otimes T^{\star} M^{k}$ are said to have weight $w+j-k$. A Weyl derivative $D$ is a connection on the density bundle. A conformal metric $c$ is a metric on the weightless tangent bundle $L^{-1} T M$ satisfying the normalizing condition $|\operatorname{det} c|=1$. This can also be considered as a metric on $T M$ with values in $L^{2}$. 
Definition 2.1. The triple $(M, c, D)$ is called a Weyl Manifold.

The bundle $L$ is trivial, and a nowhere zero section of $L, \mu$, is called a length scale. This defines a metric in the conformal class $c$ by $g_{\mu}=\mu^{-2} c$. The section $\mu$ gives a trivialization of $L$ which has a corresponding trivializing connection $D^{\mu}$. This defines a one form $\alpha_{\mu}=D-D^{\mu}$, so that

$$
D(h \mu)=(d h+h D) \mu=\left(d h+h\left(\alpha_{\mu}+D^{\mu}\right)\right) \mu=\left(d h+h \alpha_{\mu}\right) \mu .
$$

There is a unique torsion free connection $\nabla^{D}$ on TM making $c$ parallel,

$$
\nabla_{X}^{D} Y=\nabla_{X}^{g_{\mu}} Y+\alpha_{\mu}(X) Y+\alpha_{\mu}(Y) X-g_{\mu}(X, Y) \alpha_{\mu}^{\sharp g_{\mu}},
$$

where $\nabla^{g_{\mu}}$ is the Levi-Civita connection for the metric $g_{\mu}$.

\subsubsection{Weightless Twistor Space}

When $M$ is oriented, $c$ defines a section $v_{c}$ of the orientation bundle $L^{n} \Lambda^{n} T^{\star} M$. This can be used to define the conformal Hodge star

$$
{ }^{\star}: L^{m} \Lambda^{k} T^{\star} M \rightarrow L^{m+n-2 k} \Lambda^{n-k} T^{\star} M,
$$

where for $\beta, \gamma \in L^{k} \Lambda^{k} T^{\star} M$

$$
\beta \wedge{ }^{\star} \gamma=c(\beta, \gamma) v_{c}
$$

For $n=4$ and $m=0$, * $: \Lambda^{2} T^{\star} M \rightarrow \Lambda^{2} T^{\star} M$ is an involution with \pm 1 eigenspaces $\Lambda_{ \pm}^{2} T^{\star} M$. The weightless twistor spaces [2] can be constructed as the sphere bundles

$$
z_{ \pm}=S\left(L^{2} \Lambda_{ \pm}^{2} T^{\star} M\right)
$$

We now review the construction of an almost-complex structure $\mathcal{J}_{ \pm}$on $z_{ \pm}$. This can be seen by working at a point $q_{ \pm} \in z_{ \pm}$which projects to $p \in M$. For $U$ a neighborhood of $p$, and a local section $s_{ \pm}: U \rightarrow z_{ \pm \mid U}$ satisfying $s_{ \pm}(p)=q_{ \pm}$, there is a weightless Kähler form $\sigma_{ \pm}$given by this section and a corresponding almostcomplex structure $J_{ \pm}$on $T_{p} M$ given by

$$
\sigma_{ \pm}(X, Y)=c\left(J_{ \pm} X, Y\right) .
$$

As the fiber of $z_{ \pm}$at $p$ is a sphere in $L^{2} \Lambda_{ \pm}^{2} T_{p}^{\star} M$, the vertical tangent space at $q_{ \pm}$is the space perpendicular to $\sigma_{ \pm}$in $L^{2} \Lambda_{ \pm}^{2} T_{p}^{\star} M$. This is the space of weightless $J_{ \pm}$-anti-invariant 2-forms [4].

Definition 2.2. The space of weightless $J_{ \pm}$-anti-invariant 2-forms $L^{2} \Lambda_{-}^{2, J_{ \pm}} T_{p}^{\star} M$ is the (-1)-eigenspace for the involution $I_{ \pm}: L^{2} \Lambda^{2} T_{p}^{\star} M \rightarrow L^{2} \Lambda^{2} T_{p}^{\star} M$ given by $\left(I_{ \pm} \beta\right)(X, Y)=\beta\left(J_{ \pm} X, J_{ \pm} Y\right)$.

There is an induced almost-complex structure acting on $\beta \in L^{2} \Lambda_{-}^{2, J_{ \pm}} T_{p}^{\star} M$ by

$$
\left(J_{ \pm} \beta\right)(X, Y)=\beta\left(J_{ \pm} X, Y\right) .
$$

To see that this is an almost-complex strucure, first note that $J_{ \pm} \beta$ is a two form as

$$
\beta\left(J_{ \pm} X, Y\right)=-\beta\left(Y, J_{ \pm} X\right)=\beta\left(J_{ \pm} Y, J_{ \pm}^{2} X\right)=-\beta\left(J_{ \pm} Y, X\right) .
$$

Second,

$$
I_{ \pm} J_{ \pm} \beta=J_{ \pm} I_{ \pm} \beta=-J_{ \pm} \beta,
$$

so $J_{ \pm} \beta \in L^{2} \Lambda_{-}^{2, J_{ \pm}} T_{p}^{\star} M$. Finally, it is easily seen that $J_{ \pm}^{2} \beta=-\beta$.

Extending $\beta$ to be complex bilinear gives

$$
\begin{aligned}
& \frac{1}{4} \beta\left(X+i J_{ \pm} X, Y+i J_{ \pm} Y\right)=\frac{1}{2}\left(\beta+i J_{ \pm} \beta\right)(X, Y), \\
& \frac{1}{4} \beta\left(X-i J_{ \pm} X, Y+i J_{ \pm} Y\right)=0 \\
& \frac{1}{4} \beta\left(X-i J_{ \pm} X, Y-i J_{ \pm} Y\right)=\frac{1}{2}\left(\beta-i J_{ \pm} \beta\right)(X, Y) .
\end{aligned}
$$


Therefore $\beta \in L^{2}\left(\Lambda_{ \pm}^{2,0} T_{p}^{\star} M \oplus \Lambda_{ \pm}^{0,2} T_{p}^{\star} M\right)$. This shows that $L^{2} \Lambda_{-}^{2, J_{ \pm}} T_{p}^{\star} M \perp \sigma_{ \pm}$as there is an orthogonal splitting

$$
\Lambda_{ \pm}^{2} T_{p}^{\star} M \otimes \mathbb{C}=\Lambda_{ \pm}^{2,0} T_{p}^{\star} M \oplus \Lambda_{ \pm}^{0,2} T_{p}^{\star} M \oplus \mathbb{C} \sigma_{ \pm} .
$$

Furthermore $\frac{1}{2}\left(\beta-i J_{ \pm} \beta\right) \in L^{2} \Lambda_{ \pm}^{2,0} T_{p}^{\star} M$ and $\frac{1}{2}\left(\beta+i J_{ \pm} \beta\right) \in L^{2} \Lambda_{ \pm}^{0,2} T_{p}^{\star} M$.

There is an isomorphism, $\beta \mapsto \beta^{v}$, from $L^{2} \Lambda_{-}^{2, J_{ \pm}} T_{p}^{\star} M$ to the vertical tangent space $V\left(T_{q_{ \pm}} z_{ \pm}\right)$, so that for $\beta \in L^{2} \Lambda_{-}^{2, J_{ \pm}} T_{p}^{\star} M$ we have $\beta^{v} \in V\left(T_{q_{ \pm}} z_{ \pm}\right)$. This isomorphism and the connection induce an isomorphism, $X \mapsto X^{h}$, from $T_{p} M$ to the horizontal tangent space $H\left(T_{q_{ \pm}} z_{ \pm}\right)$by

$$
d s_{ \pm}(X)=X^{h}+\left(\nabla_{X}^{D} \sigma_{ \pm}\right)^{v} .
$$

The almost-complex structure on $z_{ \pm}$is now given by linearly extending

$$
\begin{aligned}
\mathcal{J}_{ \pm}\left(X^{h}\right) & :=\left(J_{ \pm} X\right)^{h}, \\
\mathcal{J}_{ \pm}\left(\beta^{v}\right) & :=\left(J_{ \pm} \beta\right)^{v} .
\end{aligned}
$$

In [5] Eells and Salamon used a similar complex structure to study weakly conformal harmonic maps. Their complex structure is the same as the complex structure of Penrose, studied by Atiyah, Hitchen and Singer in [1], except that it reverses the orientation of the fibers. The complex structure defined in (5) and (6) differs from that of Eells and Salamon only in the use of a Weyl connection to define the horizontal space rather than the Levi-Civita connection.

\subsubsection{Submanifold Geometry}

Let $(M, c, D)$ be a Weyl manifold, and $i: \Sigma \rightarrow M$ an immersed submanifold. Then $\Sigma$ inherits a conformal structure $\bar{c}$ and Weyl derivative $\bar{D}$. One way to see this is to choose a length scale, $\mu \in \Gamma(L)$. Then the metric $g_{\mu}$ and the one form $\alpha_{\mu}$ can be pulled back to $\Sigma$ as $\bar{g}_{\mu}=i^{\star} g_{\mu}$ and $\bar{\alpha}_{\mu}=i^{\star} \alpha_{\mu}$. Hence $\bar{\mu}=\left|\operatorname{det} \bar{g}_{\mu}\right|^{-1 /(2 \operatorname{dim} \Sigma)}$ is a section of the density bundle of $\Sigma$. The inherited conformal metric is $\bar{c}=\bar{\mu}^{2} \bar{g}_{\mu}$ and the inherited Weyl derivative is

$$
\bar{D}(h \bar{\mu})=\left(d h+h \bar{\alpha}_{\mu}\right) \bar{\mu} .
$$

The connection $\bar{\nabla}^{\bar{D}}$ on $\Sigma$ is defined so that $\bar{c}$ is parallel,

$$
\bar{\nabla}_{X}^{\bar{D}} Y=\bar{\nabla}_{X}^{\bar{g}_{\mu}} Y+\bar{\alpha}_{\mu}(X) Y+\bar{\alpha}_{\mu}(Y) X-\langle X, Y\rangle_{\bar{g}_{\mu}} \bar{\alpha}_{\mu}^{\sharp \bar{s}_{\mu}} .
$$

The Weyl second fundamental form [13] is given by

$$
B^{D}(X, Y)=\nabla_{X}^{D} Y-\bar{\nabla}_{X}^{\bar{D}} Y .
$$

Equivalently, for $A_{g_{\mu}}=\nabla^{g_{\mu}}-\bar{\nabla}^{\bar{g}_{\mu}}$

$$
B^{D}(X, Y)=A_{g_{\mu}}(X, Y)-\langle X, Y\rangle_{g_{\mu}}\left(\alpha_{\mu}^{\sharp g_{\mu}}\right)^{\perp} .
$$

The Weyl mean curvature is

$$
\mathbf{H}^{D}=\frac{1}{\operatorname{dim} \Sigma} \operatorname{tr}_{\bar{g}_{\mu}} B^{D}=\mathbf{H}_{g_{\mu}}-\left(\alpha_{\mu}^{\sharp g_{\mu}}\right)^{\perp},
$$

where $\mathbf{H}_{g_{\mu}}$ is the usual mean curvature of $\Sigma$ with respect to the metric $g_{\mu}$.

Definition 2.3. The immersion $i: \Sigma \rightarrow M$ is Weyl-minimal if $\mathbf{H}^{D}=0$.

Example 2.1. Exact and Closed Weyl Derivatives

If $(M, c, D)$ is a Weyl manifold and there is a length scale $\mu$ so that $\alpha_{\mu}$ is exact, then $D$ is called exact. If $\alpha_{\mu}=d u$, then $D=D^{e^{-u} \mu}$ and the Weyl-minimal surfaces are just the minimal surfaces for the metric $g^{e^{-u} \mu}=e^{2 u} g^{\mu}$. Similarly, if $\alpha_{\mu}$ is closed then $D$ is called closed. In this case, if $f: \Sigma \rightarrow M$ is a Weyl-minimal branched immersion then there is a lift to the universal cover $\tilde{f}: \tilde{\Sigma} \rightarrow \tilde{M}$. The conformal metric and Weyl Derivative can be lifted to $\tilde{M}$ and the closed Weyl derivative becomes exact. Thus $\tilde{f}$ is a minimal surface for a metric in the lifted conformal class. 
The harmonic map equation can also be generalized to this setting. In [8] the second fundamental form of a map $f: \Sigma \rightarrow M$ is defined for manifolds $\Sigma$ and $M$ with torsion-free connections $\nabla^{\Sigma}$ and $\nabla^{M}$. If $\nabla$ is the induced connection on $T^{\star} \Sigma \otimes f^{\star} T M$, then the second fundamental form is just $\nabla d f$. If $\eta$ is a metric on $\Sigma$ then the tension of the map can be defined as

$$
\tau\left(\eta, \nabla^{\Sigma}, \nabla^{M}\right)=\operatorname{tr}_{\eta} \nabla d f .
$$

A map is psuedo-harmonic if the tension field is zero. We study the case where the domain $(\Sigma, \eta)$ is a Riemannian manifold with its Levi-Civita connection $\nabla^{\eta}$ and the target manifold $(M, c, D)$ is a Weyl manifold. This is opposite of the case studied in [8], where the domain is Weyl and the target is Riemannian.

Definition 2.4. A map $f: \Sigma \rightarrow M$ is Weyl-harmonic if $\tau\left(\eta, \nabla^{\eta}, \nabla^{D}\right)=0$.

From this point we only consider the case where $\Sigma$ has dimension two. Using local isothermal coordinates on $\Sigma$ so that $\eta=e^{2 \lambda}\left(d x^{2}+d y^{2}\right)$, the tension field is

$$
\tau\left(\eta, \nabla^{\eta}, \nabla^{D}\right)=e^{-2 \lambda}\left(\nabla_{\partial_{x}}^{D} f_{x}+\nabla_{\partial_{y}}^{D} f_{y}\right),
$$

where $f_{x}=d f\left(\partial_{x}\right)$. In terms of the complex coordinate $z=x+i y$ this is just

$$
\tau\left(\eta, \nabla^{\eta}, \nabla^{D}\right)=4 e^{-2 \lambda} \nabla_{\partial_{\bar{z}}}^{D} f_{z}
$$

This can also be written more explicitly as

$$
\tau\left(\eta, \nabla^{\eta}, \nabla^{D}\right)=e^{-2 \lambda}\left(\nabla_{\partial_{x}}^{g_{\mu}} f_{x}+\nabla_{\partial_{y}}^{g_{\mu}} f_{y}+2 \alpha_{\mu}\left(f_{x}\right) f_{x}+2 \alpha_{\mu}\left(f_{y}\right) f_{y}-\left(\left|f_{x}\right|_{g_{\mu}}^{2}+\left|f_{y}\right|_{g_{\mu}}^{2}\right) \alpha_{\mu}^{\sharp g_{\mu}}\right) .
$$

From this we see that when $\Sigma$ has dimension 2

$$
\tau\left(e^{2 u} \eta, \nabla^{2 u} \eta, \nabla^{D}\right)=e^{-2 u} \tau\left(\eta, \nabla^{\eta}, \nabla^{D}\right) .
$$

Thus, in this case, the definition of Weyl-harmonic depends only on the conformal class of $\eta$. We are also interested in the case where $f$ is weakly conformal.

Definition 2.5. A map $f: \Sigma \rightarrow M$ is weakly conformal if it is conformal whenever $d f \neq 0$.

If $z=x+i y$ is a complex coordinate on $\Sigma$ so that $\eta=e^{2 \lambda}\left(d x^{2}+d y^{2}\right)$, then the equations

$$
\eta\left(\partial_{x}, \partial_{y}\right)=0 \quad \text { and } \quad \eta\left(\partial_{x}, \partial_{x}\right)=\eta\left(\partial_{y}, \partial_{y}\right)
$$

are conformally invariant. If $f$ is weakly conformal then this implies that

$$
c\left(f_{x}, f_{y}\right)=0 \quad \text { and } \quad c\left(f_{x}, f_{x}\right)=c\left(f_{y}, f_{y}\right) .
$$

Extending the conformal inner product to be complex bilinear, these are equivalent to the equation

$$
c\left(f_{z}, f_{z}\right)=0
$$

where $f_{z}=\frac{1}{2}\left(f_{x}-i f_{y}\right)$. Any point where $d f$ is not full rank is called a singular point. A branch point $p$ is a singular point where in some neighborhood of $p, f_{z}=z^{k} Z$ and $Z_{p} \neq 0$.

Proposition 2.1. If $f: \Sigma \rightarrow M$ is weakly conformal, Weyl-harmonic, and non-constant, then $d f$ is rank 2 except at an isolated set of branch points.

Proof. Like the harmonic map equation, the Weyl-harmonic map equation can be written as the Laplace equation plus terms quadratic in $d f$. Thus the hypotheses of Aronzajn's unique continuation theorem and the Hartman-Wintner theorem [11] are still satisfied in the Weyl-harmonic case. As $f$ is non-constant, Aronzajn's 
theorem implies that $d f$ is not zero on an open set. By the Hartman-Wintner Theorem, at every point there is an integer $m \geq 1$ so that in an isothermal coordinate chart,

$$
f(x, y)=h(x, y)+o\left(|(x, y)|^{m}\right) \quad \text { and } \quad d f(x, y)=d h(x, y)+o\left(|(x, y)|^{m-1}\right) .
$$

for some non-zero homogeneous degree $m$ polynomial $h$. The zeros of $d h$ are isolated, so the zeros of $d f$ must be as well. In fact, $h$ must also be weakly conformal and harmonic, thus the only zeros of $d h$ are branch point singularities. Details and further analysis of the structure of these branch points is contained in [12].

If $f: \Sigma \rightarrow M$ is an immersion with only branch point singularities, then it is called a branched immersion. When $f$ is a branched immersion the conformal class $f^{\star} c$ on $\Sigma$ can be defined across the branch points and $f$ is weakly conformal when $f^{\star} c=[\eta]$. A branched immersion which is Weyl-minimal away from the branch points is called a branched Weyl-minimal immersion.

Proposition 2.2. If $f: \Sigma \rightarrow M$ is weakly conformal then $f$ is Weyl-harmonic if and only if it is a branched Weyl-minimal immersion.

Proof. In this case, away from the branch points,

$$
\tau\left(\eta, \nabla^{\eta}, \nabla^{D}\right)=e^{-2 \lambda}\left(\left|f_{x}\right|_{g_{\mu}}^{2}+\left|f_{y}\right|_{g_{\mu}}^{2}\right)\left(\mathbf{H}_{g_{\mu}}-\left(\alpha_{\mu}^{\sharp g_{\mu}}\right)^{\perp}\right) .
$$

Comparing this with equation (7) we see that $\tau\left(\eta, \nabla^{\eta}, \nabla^{D}\right)=0$ if and only if $\mathbf{H}^{D}=0$.

There is a splitting $T M=T_{f} \Sigma \oplus N_{f} \Sigma$, where $T_{f} \Sigma=d f(T \Sigma)$ away from the branch points. At a branch point $p$, $f_{z}=z^{k} Z, Z_{p} \neq 0$, and $T_{f} \Sigma=\operatorname{span}\left\{\operatorname{Re}\left(Z_{p}\right), \operatorname{Im}\left(Z_{p}\right)\right\}$. In both cases $N_{f} \Sigma$ is the orthogonal complement of $T_{f} \Sigma$.

Definition 2.6. For $M$ oriented, the twistor lifts of $f, \tilde{f}_{ \pm}: \Sigma \rightarrow z_{ \pm}$are determined by two complex structures on $f^{\star} T M$. There are two orthogonal complex structures $J_{ \pm}$which agree with the complex structure of $\Sigma$ on $T_{f} \Sigma$. The complex structure $J_{+}$preserves the orientation while $J_{-}$is orientation reversing. The corresponding weightless Kähler form is then

$$
\tilde{f}_{ \pm}=c\left(J_{ \pm} \cdot, \cdot\right)
$$

The complex structures $J_{ \pm}$determine a splitting of $f^{\star} T M \otimes \mathbb{C}=f^{\star} T_{ \pm}^{(1,0)} M \oplus f^{\star} T_{ \pm}^{(0,1)} M$.

\subsection{Conformally Almost-Hermitian Manifolds}

A conformally almost-Hermitian manifold $\left(M^{4}, c, J\right)$ is an almost-complex manifold with a conformal structure satisfying

$$
c(X, Y)=c(J X, J Y) .
$$

The conformal Kähler form $\omega_{c}=c(J \cdot, \cdot)$ can be viewed as a 2-form with values in $L^{2}$. Then there is a unique Weyl derivative satisfying $d^{D} \omega_{c}=0$. Fixing $\mu \in \Gamma(L)$ we can define this Weyl derivative using the Lee form

$$
\theta_{\mu}=J \delta_{g_{\mu}} \omega_{\mu}=-\left(\delta_{g_{\mu}} \omega_{\mu}\right) J,
$$

with $\delta_{g_{\mu}}$ denoting the divergence and $\omega_{c}=\mu^{2} \omega_{\mu}$. In terms of an orthonormal coframe $\left\{e^{i}\right\}$ of $g_{\mu}$ satisfying

$$
\omega_{\mu}=e^{1} \wedge e^{2}+e^{3} \wedge e^{4},
$$

and $a_{i}=\left\langle e_{i}, \delta_{g_{\mu}} \omega_{\mu}\right\rangle_{g_{\mu}}$ one can check that

$$
d \omega_{\mu}=a_{1} e^{2} \wedge e^{3} \wedge e^{4}-a_{2} e^{1} \wedge e^{3} \wedge e^{4}+a_{3} e^{1} \wedge e^{2} \wedge e^{4}-a_{4} e^{1} \wedge e^{2} \wedge e^{3} .
$$

Then the Lee form is

$$
\theta_{\mu}=-J^{\star} d \omega_{\mu}=J\left(a_{1} e^{1}+a_{2} e^{2}+a_{3} e^{3}+a_{4} e^{4}\right)=a_{1} e^{2}-a_{2} e^{1}+a_{3} e^{4}-a_{4} e^{3},
$$


furthermore

$$
\theta_{\mu} \wedge \omega_{\mu}=d \omega_{\mu}
$$

Then for Weyl derivative $D=d+\alpha_{\mu}$,

$$
d^{D} \omega=d^{D} \mu^{2} \omega_{\mu}=2 \mu D(\mu) \omega+\mu^{2} d \omega=2 \mu^{2} \alpha_{\mu} \wedge \omega_{\mu}+\mu^{2} \theta_{\mu} \wedge \omega_{\mu} .
$$

The canonical Weyl derivative of $(M, c, J)$ is then determined by setting $\alpha_{\mu}=-\frac{1}{2} \theta_{\mu}$. The induced connection on $T M$ is given by

$$
\nabla_{X}^{D} Y=\nabla_{X}^{g_{\mu}} Y-\frac{1}{2} \theta_{\mu}(X) Y-\frac{1}{2} \theta_{\mu}(Y) X+\frac{1}{2}\langle X, Y\rangle_{g_{\mu}} \theta_{\mu}^{\sharp g_{\mu}} .
$$

The Nijenhuis Tensor of $J$ is given by

$$
N(X, Y)=[X, Y]+J[J X, Y]+J[X, J Y]-[J X, J Y] .
$$

This is $J$ antilinear in both slots, that is $N(J X, Y)=-J N(X, Y)=N(X, J Y)$. For any vector field, $X, \nabla_{X}^{D} J$ is also $J$ antilinear.

Proposition 2.3. For any almost-Hermitian manifold

$$
\langle N(X, Y), J Z\rangle_{g_{\mu}}=d \omega_{\mu}(X, Y, Z)-d \omega_{\mu}(J X, J Y, Z)-2\left\langle\left(\nabla_{Z}^{g_{\mu}} J\right) X, Y\right\rangle_{g_{\mu}} .
$$

This is proposition 4.2 in [7] with different conventions. The corresponding formula for conformally almostHermitian manifolds with a Weyl derivative is

$$
c(N(X, Y), J Z)=d^{D} \omega_{c}(X, Y, Z)-d^{D} \omega_{c}(J X, J Y, Z)-2 c\left(\left(\nabla_{Z}^{D} J\right) X, Y\right) .
$$

Proposition 2.4. For any conformally almost-Hermitian manifold with canonical Weyl derivative $D$,

$$
c(N(X, Y), J Z)=-2 c\left(\left(\nabla_{Z}^{D} J\right) X, Y\right) .
$$

Corollary 2.1. The global section $s:(M, J) \rightarrow\left(\mathcal{Z}_{+}, \mathcal{J}_{+}\right)$defined by $\omega_{c}$ is holomorphic.

Proof. Letting $X, Y, Z \in T M$, by the proposition,

$$
\left(\nabla_{Z}^{D} \omega_{c}\right)(X, Y)=-\frac{1}{2} c(N(X, Y), J Z)
$$

and by the symmetries of the Nijenhuis tensor,

$$
\left(\nabla_{J Z}^{D} \omega_{c}\right)(X, Y)=\frac{1}{2} c(N(X, Y), Z)=\frac{1}{2} c(J N(X, Y), J Z)=-\frac{1}{2} c(N(J X, Y), J Z) .
$$

Therefore $\left(\nabla_{J Z}^{D} \omega_{c}\right)=\mathcal{J}_{+}\left(\nabla_{Z}^{D} \omega_{c}\right)$, and by equations (4), (5), and (6)

$$
d s(J Z)=(J Z)^{h}+\left(\nabla_{J Z}^{D} \omega_{c}\right)^{v}=\mathcal{J}_{+} Z^{h}+\mathcal{J}_{+}\left(\nabla_{Z}^{D} \omega_{c}\right)^{v}=\mathcal{J}_{+} d s(Z) .
$$

\section{Main Theorems}

\subsection{Twistor Correspondence}

Following [5] we now show there is a correspondence between weakly conformal Weyl-harmonic maps and non-vertical $\mathcal{J}_{ \pm}$-holomorphic maps into the weightless twistor space with complex structure given by (5) and (6). 
The twistor lifts of a weakly conformal map $f: \Sigma \rightarrow M$ are given by

$$
\tilde{f}_{ \pm}=\left(\frac{\left(1 \pm^{\star}\right) f_{z} \wedge f_{\bar{z}}}{i c\left(f_{z}, f_{\bar{z}}\right)}\right)^{b_{c}},
$$

where $i=\sqrt{-1}$. The natural isomorphism $b_{c}: L^{-1} T M \rightarrow L T^{\star} M$ preserves weights, but interchanges the holomorphic and anti-holomorphic spaces.

Theorem 3.1. For any Weyl manifold $(M, c, D)$, a weakly conformal map $f: \Sigma \rightarrow M$ is Weyl-harmonic if and only if the twistor lifts $\tilde{f}_{ \pm}: \Sigma \rightarrow z_{ \pm}$are $\mathcal{J}_{ \pm}$-holomorphic.

Proof. The twistor lifts $\tilde{f}_{ \pm}$are $\mathcal{J}_{ \pm}$-holomorphic provided $d \tilde{f}_{ \pm}\left(\partial_{z}\right) \in T^{(1,0)} z_{ \pm}$. We have

$$
d \tilde{f}_{ \pm}\left(\partial_{z}\right)=\left(f_{z}\right)^{h}+\left(\nabla_{\partial_{z}}^{D} \tilde{f}_{ \pm}\right)^{v}
$$

and since $f_{z} \in T_{ \pm}^{(1,0)} M$, we have $\left(f_{z}\right)^{h} \in H\left(T^{(1,0)} z_{ \pm}\right)$. Thus all that is required is $\nabla_{\partial}^{D} \tilde{f}_{ \pm} \in L^{2} \Lambda^{(2,0)} T^{\star} M$. We find that

$$
\begin{aligned}
& \left(\nabla_{\partial_{z}}^{D} \tilde{f}_{ \pm}\right)_{ \pm}^{(0,2)}=\left(\frac{\sqrt{2} f_{z} \wedge\left(\nabla_{\partial_{z}}^{D} f_{\bar{z}}\right)_{ \pm}^{(1,0)}}{i c\left(f_{z}, f_{\bar{z}}\right)}\right)^{b_{c}}, \\
& \left(\nabla_{\partial_{z}}^{D} \tilde{f}_{ \pm}\right)_{ \pm}^{(1,1)}=0, \\
& \left(\nabla_{\partial_{z}}^{D} \tilde{f}_{ \pm}\right)_{ \pm}^{(2,0)}=\left(\frac{\sqrt{2}\left(\nabla_{\partial_{z}}^{D} f_{z}\right)_{ \pm}^{(0,1)} \wedge f_{\bar{z}}}{i c\left(f_{z}, f_{\bar{z}}\right)}\right)^{b_{c}} .
\end{aligned}
$$

It follows that $\tilde{f}_{ \pm}$is pseudo-holomorphic map if and only if $\left(\nabla_{\partial_{z}}^{D} f_{\bar{z}}\right)_{ \pm}^{(1,0)}=k f_{z}$, for some function $k$. Taking the conformal inner-product with $f_{\bar{z}}$ gives

$$
c\left(\left(\nabla_{\partial_{z}}^{D} f_{\bar{z}}\right)_{ \pm}^{(1,0)}, f_{\bar{z}}\right)=k c\left(f_{z}, f_{\bar{z}}\right)
$$

Since $f_{\bar{z}} \in T_{ \pm}^{(0,1)} M$ this is just

and since $c$ is $\nabla^{D}$ parallel we have

$$
c\left(\nabla_{\partial_{z}}^{D} f_{\bar{z}}, f_{\bar{z}}\right)=k c\left(f_{z}, f_{\bar{z}}\right),
$$

$$
\nabla_{\partial_{z}}^{D} c\left(f_{\bar{z}}, f_{\bar{z}}\right)=2 k c\left(f_{z}, f_{\bar{z}}\right) .
$$

For $f$ weakly conformal, this shows that $k=0$. Therefore $\tilde{f}_{ \pm}$is $\mathcal{J}$-holomorphic if and only if $\left(\nabla_{\partial_{z}}^{D} f_{\bar{z}}\right)_{ \pm}^{(1,0)}=0$, but since $\nabla_{\partial_{z}}^{D} f_{\bar{z}}$ is real, this can only be true when it is zero.

Corollary 3.1. There is a one-to-one correspondence between weakly conformal Weyl-harmonic maps to $(M, c, D)$ and non-vertical $\mathcal{J}_{ \pm}$-holomorphic maps to the twistor space.

Proof. It only remains to show that for a non-vertical $\mathcal{J}$-holomorphic curve, $\phi: \Sigma \rightarrow z_{ \pm}$the projection $\bar{\phi}$ : $\Sigma \rightarrow M$ is weakly conformal and Weyl-harmonic. It is clearly weakly conformal as $\bar{\phi}_{z}$ is holomorphic with respect to the complex structure defined by $\phi$, which implies $c\left(\bar{\phi}_{z}, \bar{\phi}_{z}\right)=0$. It is Weyl-harmonic as $\phi$ is its twistor lift and is J-holomorphic.

Corollary 3.2. The J-holomorphic curves $f: \Sigma \rightarrow M$ are weakly conformal and Weyl-harmonic.

Proof. The composition with the section $s: M \rightarrow z_{+}$determined by $J$ is a $\mathcal{J}_{+}$-holomorphic curve of $z_{+}$.

\subsection{Adjunction Inequality}

In this section we prove theorem 1.3 
Proof. Fix a metric $\langle$,$\rangle in the conformal class. For a holomorphic normal coordinate z$ on $\Sigma$, split $f_{z}$ into its holomorphic and antiholomorphic parts

$$
\alpha=\frac{1}{2}\left(f_{z}-i J f_{z}\right) \quad \bar{\beta}=\frac{1}{2}\left(f_{z}+i J f_{z}\right),
$$

we have

$$
\langle\alpha, \alpha\rangle=0=\langle\beta, \beta\rangle .
$$

When $f$ is weakly conformal, this implies that

$$
\langle\alpha, \bar{\beta}\rangle=0 .
$$

Thus $\alpha$ and $\beta$ are Hermitian orthogonal and away from their zeros span the holomorphic tangent bundle $f^{\star} T^{(1,0)} M$. The Weyl-harmonic map equation in coordinates is

$$
\nabla_{\partial_{\bar{z}}}^{D} f_{z}=0
$$

Since $\nabla^{D}$ does not preserve the almost-complex structure, we write the equation using the connection $\nabla^{D, J}$, given by

$$
\nabla_{X}^{D, J} Y=\nabla_{X}^{D} Y-\frac{1}{2} J\left(\nabla_{X}^{D} J\right) Y
$$

This connection preserves the complex structure, and thus preserves the holomorphic and anti-holomorphic tangent spaces. In terms of this connection the Weyl-harmonic map equation is

$$
\nabla_{\partial_{\bar{z}}}^{D, J} \frac{\partial \phi}{\partial z}=-\frac{1}{2} J\left(\nabla_{\partial_{\bar{z}}}^{D} J\right) \frac{\partial \phi}{\partial z} .
$$

Since $\nabla_{\partial_{\bar{z}}}^{D} J$ is $J$ anti-linear, it maps from $T^{(1,0)} M$ to $T^{(0,1)} M$ and from $T^{(0,1)} M$ to $T^{(1,0)} M$. The Weyl-harmonic map equation can then be written in terms of $\alpha$ and $\bar{\beta}$ as

$$
\begin{aligned}
& \nabla_{\partial_{\bar{z}}}^{D, J} \alpha=-\frac{i}{2}\left(\nabla_{\partial_{\bar{z}}}^{D} J\right) \bar{\beta}, \\
& \nabla_{\partial_{\bar{z}}}^{D, J} \bar{\beta}=\frac{i}{2}\left(\nabla_{\partial_{\bar{z}}}^{D} J\right) \alpha .
\end{aligned}
$$

Using proposition 2.4 , a weakly conformal Weyl-harmonic map must satisfy

$$
\begin{aligned}
\left\langle\nabla_{\partial_{\bar{z}}}^{D, J} \alpha, \bar{\alpha}\right\rangle & =-\frac{i}{2}\left\langle\left(\nabla_{\partial_{\bar{z}}}^{D} J\right) \bar{\beta}, \bar{\alpha}\right\rangle, & \left\langle\nabla_{\partial_{\bar{z}}}^{D, J} \alpha, \bar{\beta}\right\rangle & =-\frac{i}{2}\left\langle\left(\nabla_{\partial_{\bar{z}}}^{D} J\right) \bar{\beta}, \bar{\beta}\right\rangle, \\
& =\frac{i}{4}\left\langle N(\bar{\beta}, \bar{\alpha}), J f_{\bar{z}}\right\rangle, & & =\frac{i}{4}\left\langle N(\bar{\beta}, \bar{\beta}), J f_{\bar{z}}\right\rangle, \\
& =\frac{1}{4}\langle N(\bar{\alpha}, \bar{\beta}), \bar{\alpha}\rangle, & & =0,
\end{aligned}
$$

where the last line follows from $\bar{\alpha}, \bar{\beta} \in f^{\star} T^{(0,1)} M$, which implies $N(\bar{\alpha}, \bar{\beta}) \in f^{\star} T^{(1,0)} M$. This implies that away from the zeros of $\alpha$ and $\beta$,

$$
\nabla_{\partial \bar{z}}^{D, J} \alpha=\frac{\langle N(\bar{\alpha}, \bar{\beta}), \bar{\alpha}\rangle}{4\|\alpha\|^{2}} \alpha
$$

Similarly

$$
\begin{aligned}
\left\langle\nabla_{\partial_{\bar{z}}}^{D, J} \bar{\beta}, \beta\right\rangle & =\frac{i}{2}\left\langle\left(\nabla_{\partial_{\bar{z}}}^{D} J\right) \alpha, \beta\right\rangle, & \left\langle\nabla_{\partial_{\bar{z}}}^{D, J} \bar{\beta}, \alpha\right\rangle & =\frac{i}{2}\left\langle\left(\nabla_{\partial_{\bar{z}}}^{D} J\right) \alpha, \alpha\right\rangle, \\
& =-\frac{i}{4}\left\langle N(\alpha, \beta), J f_{\bar{z}}\right\rangle, & & =-\frac{i}{4}\left\langle N(\alpha, \alpha), J f_{\bar{z}}\right\rangle, \\
& =\frac{1}{4}\langle N(\alpha, \beta), \beta\rangle, & & =0 .
\end{aligned}
$$

This gives

$$
\nabla_{\partial_{\bar{z}}}^{D, J} \bar{\beta}=\frac{\langle N(\alpha, \beta), \beta\rangle}{4\|\beta\|^{2}} \bar{\beta} .
$$


By the Koszul-Malgrange theorem [9], there are holomorphic structures on $f^{\star} T^{(1,0)} M$ and $f^{\star} T^{(0,1)} M$ so that

$$
\bar{\partial} X=\nabla_{\partial_{\bar{z}}}^{D, J} X \otimes d \bar{z}
$$

Then for a Weyl-harmonic map

$$
\begin{aligned}
& \bar{\partial} \alpha=\frac{\langle N(\bar{\alpha}, \bar{\beta}), \bar{\alpha}\rangle}{4\|\alpha\|^{2}} \alpha \otimes d \bar{z}, \\
& \bar{\partial} \bar{\beta}=\frac{\langle N(\alpha, \beta), \beta\rangle}{4\|\beta\|^{2}} \bar{\beta} \otimes d \bar{z} .
\end{aligned}
$$

The Bers-Vekua similarity principle (see [6]) implies that near any point $p \in \Sigma$ we have

$$
\alpha=\gamma_{p} e^{\sigma_{p}}, \quad \bar{\beta}=\delta_{p} e^{\tau_{p}},
$$

for some local holomorphic sections $\gamma_{p}$ of $f^{\star} T^{(1,0)} M, \delta_{p}$ of $f^{\star} T^{(0,1)} M$, and some bounded functions $\sigma_{p}, \tau_{p}$. This can be used to define the indices

$$
\begin{aligned}
& R=\sum_{f_{z}(p)=0} \operatorname{ord}_{p}\left(f_{z}\right) \geq 0, \\
& Q=\sum_{\alpha(p)=0} \operatorname{ord}_{p}\left(\gamma_{p}\right)-R \geq 0, \\
& P=\sum_{\bar{\beta}(p)=0} \operatorname{ord}_{p}\left(\delta_{p}\right)-R \geq 0 .
\end{aligned}
$$

These are the total ramification index $R$, the number of anti-complex points $Q$, and the number of complex points $P$. Following [5], these determine the degrees of the line bundles spanned by the vector valued one forms $f_{z} d z, \alpha d z$ and $\bar{\beta} d z$ respectively. If $\left[f_{z}\right],[\alpha]$, and $[\beta]$ are line bundles generated by the locally defined sections then we have

$$
\begin{aligned}
R & =-\chi(\Sigma)+c_{1}\left(\left[f_{z}\right]\right), \\
Q+R & =-\chi(\Sigma)+c_{1}([\alpha]), \\
P+R & =-\chi(\Sigma)-c_{1}([\beta]) .
\end{aligned}
$$

We also have $f^{\star} T^{(1,0)} M=[\alpha] \oplus[\beta]$, and since $\alpha$ and $\bar{\beta}$ span a negatively oriented, maximal isotropic subspace of $f^{\star} T M \otimes \mathbb{C}$ which contains $f_{z}$, it must be that $f^{\star} T_{-}^{(1,0)} M=[\alpha] \oplus[\bar{\beta}]$. Therefore we have

$$
\begin{aligned}
c_{1}\left(f^{\star} T^{(1,0)} M\right) & =Q-P, \\
c_{1}\left(f^{\star} T_{-}^{(1,0)} M\right) & =Q+P+2 R+2 \chi(\Sigma), \\
& =Q+P+2 c_{1}\left(\left[f_{z}\right]\right), \\
& =Q+P+2 \chi\left(T_{f} \Sigma\right) .
\end{aligned}
$$

Since $c_{1}\left(f^{\star} T_{-}^{(1,0)} M\right)=\chi\left(T_{f} \Sigma\right)-\chi\left(N_{f} \Sigma\right)$ we now have the Webster's formulas

$$
\begin{aligned}
c_{1}\left(f^{\star} T^{(1,0)} M\right) & =Q-P, \\
\chi\left(T_{f} \Sigma\right)+\chi\left(N_{f} \Sigma\right) & =-P-Q .
\end{aligned}
$$

Since $P$ and $Q$ are both non-negative, this implies the adjunction inequality (3) of corollary 1.2

$$
\begin{aligned}
& \chi\left(T_{f} \Sigma\right)+\chi\left(N_{f} \Sigma\right)+c_{1}\left(f^{\star} T^{(1,0)} M\right)=-2 P \leq 0, \\
& \chi\left(T_{f} \Sigma\right)+\chi\left(N_{f} \Sigma\right)-c_{1}\left(f^{\star} T^{(1,0)} M\right)=-2 Q \leq 0 .
\end{aligned}
$$




\section{Examples}

\subsection{Hopf Surfaces}

The primary Hopf surface $M=S^{1} \times S^{3}$ is fibered over $S^{2}$ with fiber $T^{2}$. The bundle projection is just the projection to the $S^{3}$ component followed by the Hopf map. There is a Hermitian structure on $M$ induced by the standard Hermitian structures on the base and fiber. The Lee form is just $d \phi$, where $\phi$ is the angle along $S^{1}$. Every fiber is $J$-holomorphic and is therefore Weyl-minimal. It is also minimal as $\theta^{\sharp}$ is tangent to the fiber.

In addition, there is a Lagrangian Weyl-minimal surface for every great circle $\gamma$ in the base $S^{2}$. To see this consider the Clifford torus in $S^{3}$ which maps to $\gamma$ under the Hopf map. This torus contains two great circles on $S^{3}$, one tangent to the fiber and one perpendicular to the fiber. The great circle perpendicular to the fiber times the product $S^{1}$ gives a Lagrangian totally geodesic $T^{2}$ to which $\theta^{\sharp}$ is tangent, and is therefore Weyl-minimal.

Since $\theta=d \phi$ is closed we can look at the universal cover $\tilde{M}=\mathbb{R} \times S^{3}$. Using $\phi$ as the coordinate on $\mathbb{R}$ the metric is just

$$
g_{\tilde{M}}=d \phi^{2}+g_{S^{3}} .
$$

Therefore the Weyl-minimal surfaces will lift to minimal surfaces of the conformal metric

$$
e^{2 \phi} g_{\tilde{M}}=e^{2 \phi} d \phi^{2}+e^{2 \phi} g_{S^{3}}=\left(d e^{\phi}\right)^{2}+e^{2 \phi} g_{S^{3}} .
$$

Using the new coordinate $r=e^{\phi}$ this is just the (incomplete) flat metric on $\mathbb{R}^{4} \backslash 0 \cong \tilde{M}$.

$$
d r^{2}+r^{2} g_{S^{3}}
$$

Any surface lifted from $M$ will be invariant under deck transformation $\phi \mapsto \phi+2 \pi$ or $r \mapsto e^{2 \pi} r$. The Weylminimal surfaces described above correspond to the planes through the origin in $\mathbb{R}^{4}$.

\section{2 $U(1) \times U(1)$ Principal Bundles over a Riemann Surface}

Let $p: M \rightarrow \Sigma$ be a $U(1) \times U(1)$ principal bundle over a Riemann surface $\Sigma$ with volume form $\omega_{\Sigma}$. If $i \beta$ is a connection form then $\beta$ is an $\mathbb{R}^{2}$-valued form with components $\beta_{1}$ and $\beta_{2}$. If $\tilde{\omega}_{\Sigma}=p^{\star} \omega_{\Sigma}$ then $d \beta=F \tilde{\omega}_{\Sigma}$ where $F=\left(F_{1}, F_{2}\right): \Sigma \rightarrow \mathbb{R}^{2}$. The Kähler form $\omega=\beta_{1} \wedge \beta_{2}+\tilde{\omega}_{\Sigma}$ has exterior derivative

$$
d \omega=\left(F_{1} \beta_{2}-F_{2} \beta_{1}\right) \wedge \tilde{\omega}_{\Sigma}=\left(F_{1} \beta_{2}-F_{2} \beta_{1}\right) \wedge \omega .
$$

Therefore the Lee form is $\theta=F_{1} \beta_{2}-F_{2} \beta_{1}$. For a constant curvature connection, this will be closed. The Hopf surface is a special case for this example where $\Sigma=S^{2}$ with the round metric, and $M$ has associated bundle $M \times \mathbb{C}^{2} / U(1) \times U(1)=\mathbb{C} \oplus \mathbf{K}$. As in that case, the fiber is always a $J$-holomorphic curve and therefore Weylminimal. If a closed geodesic on $\gamma: S^{1} \rightarrow \Sigma$ has a closed horizontal lift $\tilde{\gamma}$ and the connection has constant curvature then $\tilde{\gamma}(s) \cdot\left(e^{-i F_{2} t}, e^{i F_{1} t}\right)$ parametrizes a Lagrangian minimal torus on $M$ to which $\theta^{\sharp}$ is tangent, and thus Weyl-minimal.

\section{References}

[1] M. F. Atiyah, N. J. Hitchin, and I. M. Singer. Self-duality in four-dimensional riemannian geometry. Proceedings of the Royal Society of London. Series A, Mathematical and Physical Sciences, 362(1711):425-461, 1978.

[2] David M. J. Calderbank and Henrik Pedersen. Einstein-Weyl geometry. In Surveys in differential geometry: essays on Einstein manifolds, volume 6 of Surv. Differ. Geom., pages 387-423. Int. Press, Boston, MA, 1999.

[3] J. Chen and G. Tian. Minimal surfaces in Riemannian 4-manifolds. Geometric \& Functional Analysis GAFA, 7(5):873-916, Oct 1997.

[4] Tedi Draghici, Tian-Jun Li, and Weiyi Zhang. On the J-anti-invariant cohomology of almost complex 4-manifolds. The Quarterly Journal of Mathematics, 64(1):83-111, 122011. 
[5] James Eells and Simon Salamon. Twistorial construction of harmonic maps of surfaces into four-manifolds. Annali della Scuola Normale Superiore di Pisa - Classe di Scienze, Ser. 4, 12(4):589-640, 1985.

[6] Jürgen Jost. Two-dimensional geometric variational problems. Pure and Applied Mathematics (New York). John Wiley \& Sons, Ltd., Chichester, 1991. A Wiley-Interscience Publication.

[7] Shoshichi Kobayashi and Katsumi Nomizu. Foundations of differential geometry. Vol. II. Interscience Tracts in Pure and Applied Mathematics, No. 15 Vol. II. Interscience Publishers John Wiley \& Sons, Inc., New York-London-Sydney, 1969.

[8] Gerasim Kokarev. On pseudo-harmonic maps in conformal geometry. Proc. Lond. Math. Soc. (3), 99(1):168-194, 2009.

[9] J. L. Koszul and B. Malgrange. Sur certaines structures fibrées complexes. Archiv der Mathematik, 9(1):102-109, Apr 1958.

[10] Renyi Ma. Complex points of minimal surfaces in almost Kähler manifolds. manuscripta mathematica, 95(2):159-168, Feb 1998.

[11] Dusa McDuff and Dietmar Salamon. J-holomorphic curves and symplectic topology, volume 52 of American Mathematical Society Colloquium Publications. American Mathematical Society, Providence, RI, second edition, 2012.

[12] Mario J. Micallef and Brian White. The structure of branch points in minimal surfaces and in pseudoholomorphic curves. Annals of Mathematics, 141(1):35-85, 1995.

[13] Henrik Pedersen, Yat Sun Poon, and Andrew Swann. Einstein-Weyl deformations and submanifolds. INT. J. MATH, 7:705-719, 1995.

[14] Marina Ville. On the normal bundle of minimal surfaces in almost Kähler 4-manifolds. In Harmonic morphisms, harmonic maps, and related topics (Brest, 1997), volume 413 of Chapman \& Hall/CRC Res. Notes Math., pages 159-173. Chapman \& Hall/CRC, Boca Raton, FL, 2000.

[15] S. M. Webster. Minimal surfaces in a Kähler surface. J. Differential Geom., 20(2):463-470, 1984. 